\title{
BMJ Open Mobile technology intervention for weight loss in rural men: protocol for a pilot pragmatic randomised controlled trial
}

\author{
Christine M Eisenhauer (D , ${ }^{1}$ Fabiana Almeida Brito, ${ }^{2}$ Aaron M Yoder, ${ }^{2}$ \\ Kevin A Kupzyk, ${ }^{3}$ Carol H Pullen, ${ }^{3}$ Katherine E Salinas, ${ }^{3}$ Jessica Miller, ${ }^{1}$ \\ Patricia A Hageman ${ }^{4}$
}

To cite: Eisenhauer CM, Brito FA, Yoder AM, et al. Mobile technology intervention for weight loss in rural men: protocol for a pilot pragmatic randomised controlled trial. BMJ Open 2020;10:e035089. doi:10.1136/ bmjopen-2019-035089

- Prepublication history and additional material for this paper are available online. To view these files, please visit the journal online (http://dx.doi. org/10.1136/bmjopen-2019035089).

Received 17 October 2019 Revised 02 January 2020 Accepted 04 March 2020

Check for updates

C Author(s) (or their employer(s)) 2020. Re-use permitted under CC BY-NC. No commercial re-use. See rights and permissions. Published by BMJ.

${ }^{1}$ College of Nursing- Northern Division, University of Nebraska Medical Center, Norfolk,

Nebraska, USA

${ }^{2}$ College of Public Health, University of Nebraska Medica Center, Omaha, Nebraska, USA ${ }^{3}$ College of Nursing, University of Nebraska Medical Center, Omaha, Nebraska, USA

${ }^{4}$ College of Allied Health, University of Nebraska Medical Center, Omaha, Nebraska, USA

Correspondence to Dr Christine M Eisenhauer; ceisenhauer@unmc.edu

\section{ABSTRACT}

Introduction Men who are overweight or obese in the rural Midwestern USA are an unrepresented, at-risk group exhibiting rising rates of cardiovascular disease, poor access to preventive care and poor lifestyle behaviours that contribute to sedentary lifestyle and unhealthy diet. Self-monitoring of eating and activity has demonstrated efficacy for weight loss. Use of mobile technologies for self-monitoring eating and activity may address rural men's access disparities to preventive health resources and support weight loss. Our pilot trial will assess the feasibility and acceptability of two mobile applications for weight loss in rural men to inform a future, full-scale trial. Methods and analysis A 6-month randomised controlled trial with contextual evaluation will randomise 80 men using a 1:1 ratio to either a Mobile Technology Plus (MT+) intervention or a basic Mobile Technology (MT) intervention in rural, midlife men (aged $40-69$ years). The MT+ intervention consists of a smartphone selfmonitoring application enhanced with discussion group (Lose-It premium), short message service text-based support and Wi-Fi scale. The MT group will receive only a self-monitoring application (Lose-It basic). Feasibility and acceptability will be evaluated using number of men recruited and retained, and evaluative focus group feedback. We seek to determine point estimates and variability of outcome measures of weight loss ( $\mathrm{kg}$ and $\%$ body weight) and improved dietary and physical activity behaviours (Behavioral Risk Factor Surveillance System (BRFSS) physical activity and fruit and vegetable consumption surveys, data from Lose-It! application (kcal/ day, steps/day)). Community capacity will be assessed using standard best practice methods. Descriptive content analysis will evaluate intervention acceptability and contextual sensitivity.

Ethics and dissemination This protocol was approved by the University of Nebraska Medical Center Institutional Review Board (IRB\# 594-17-EP). Dissemination of findings will occur through ClinicalTrials.gov and publish pilot data to inform the design of a larger clinical trial.

Trial registration number NCT03329079; preresults. Protocol V.10, study completion date 31 August 2020. Roles and responsibilities funder: NIH/NINR Health Disparities Section 1R15NR017522-01.
Strengths and limitations of this study

- The combination of community-engaged approaches with a pragmatic randomised controlled trial design delivers a weight loss intervention that facilitates local buy-in and is sensitive to context.

- Components of the real-world comparator are included in the expanded, more comprehensive intervention.

- Trial available only to men with means and proficiency to own and operate a smartphone.

- This study expands previous research using mobile applications to include community tailoring of the recruitment and intervention approach specific for rural men to inform a large definitive trial.

\section{INTRODUCTION}

\section{Background and rationale}

Over 55 million American men are overweight or obese. ${ }^{1}$ Rates among midlife and older men residing in the Midwestern USA have tripled in the past 20 years, with $75.3 \%$ of all adults in the USA aged 40-59 years are overweight or obese. ${ }^{2}$ Rural men report overall poorer health than urban men. ${ }^{3}$ Their obesity status predisposes high risk for metabolic syndrome and cardiovascular disease. ${ }^{3-5}$ Historically, rural men were less likely to be overweight and obese due to the high levels of physical activity involved in agricultural occupations. ${ }^{6}$ However, the mechanisation of agriculture has shifted men's work roles to more sedentary, technology-driven lifestyles, increasing the likelihood of developing overweight/obesity. ${ }^{78}$

Mobile health applications such as messaging and other interfaces available via mobile phone have demonstrated improvement in health behaviour change for weight loss among adults, ${ }^{9-11}$ and in some hard-toreach minority populations. ${ }^{12}$ The benefit 
that mobile technologies may hold for engaging hard-toreach rural men for weight loss is unknown. In addition, there has been limited study of men's health promotion through weight loss, ${ }^{13}$ particularly rural men.

Men, when compared with women, are less likely to use weight control practices, ${ }^{14}$ attempt weight loss or participate in weight loss programmes. ${ }^{15-17}$ Poor access to weight loss resources is one reason. ${ }^{18}$ Rural men also tend to exhibit dominant masculine norms ${ }^{19}$ which view help-seeking behaviours and health promotion strategies as feminine and weak. ${ }^{5}{ }^{19}$ Health promotion activities oriented to rural men's work roles are preferred. ${ }^{20}$ Therefore, a weight loss intervention whose content is adapted to the local norms, is accessible through the privacy of a smartphone, and communicated in an acceptable tone is critical. The Rural Men's Health Study plans to address the current gaps in knowledge by delivering a contextually sensitive weight loss intervention that is feasible to the rural environment and acceptable to participants.

\section{Aims}

We aim to 1) determine the feasibility and acceptability of a mobile technology enhanced self-monitoring intervention (Mobile Technology Plus (MT+)) for achieving weight loss in routine care of overweight and obese men in rural communities, 2) determine the point estimates and variability of outcome measures at 3 and 6 months following MT+ and MT interventions for achieving weight loss and improved dietary and physical activity behaviours for sample size estimation for a larger trial and 3) determine quantitative and qualitative indicators of community capacity (resource mobilisation, partnership linkages) to support a relevant weight loss intervention for rural men.

\section{METHODS AND ANALYSIS Design overview}

We propose a pilot, feasibility, ${ }^{21}$ pragmatic randomised controlled trial (pRCT) with an allocation ratio of 1:1. We will randomise 80 men into two groups: intervention and comparison. This pRCT will observe men in real-life rural conditions using varied versions of a mobile phone-based self-monitoring application: one that is free and available in the community setting and an enhanced, premium version..$^{22}$ Recommendations of good practice for design and analysis of a pilot study note that 30 participants per group is sufficient to estimate values for future trials sample size calculations. ${ }^{23}{ }^{24}$ As weight loss studies in rural men are a gap in the current literature, it is important that we obtain reliable estimates of effect size with which to perform a power analysis for subsequent research. So 30 participants per group will be needed, for a total of 60 . Some participants will likely not complete the study, so we will enrol 80 men to allow for up to a $25 \%$ attrition rate. Participants will complete assessments at baseline, 3 and 6 months postbaseline.

\section{Public and patient involvement}

Community engagement was used to inform the development of this protocol involving health professions students from the disciplines of nursing, physical therapy and public health. A community advisory board (CAB) was also developed. The $\mathrm{CAB}$ members represent the rural sampling region (ie, farmers, insurance and machinery dealers, extension staff and community health workers) and meet quarterly to inform the study approach, material content and imaging, targeted venues for social marketing, dissemination of recruitment materials and the direct referral of eligible participants. The funding source for this pilot study requires student involvement in study activities. One goal of the supporting grant mechanism was to expose under-represented rural students to research. Therefore, we involved graduate and undergraduate student nurses from the study sampling region. ${ }^{25}$ We are involving undergraduate level student nurses in planning, implementation and evaluation of community outreach and recruitment strategies. Two graduate (Masters and $\mathrm{PhD}$ ) level student nurses, who are part of the investigative team, will also be used to assist with implementation and evaluation of the intervention, as described in Participants and interventions.

\section{Participants and interventions \\ Participant eligibility}

Inclusion criteria: 1) man age 40-69 years, 2) reside (majority of the time) in Northeast Nebraska, USA (RUCA code 4-10), 3) body mass index (BMI) of $28(\mathrm{~kg} /$ $\mathrm{m}^{2}$ ) or higher and weight not $>396$ pounds (a man with a BMI of 50 or higher will require clinician clearance), 4) smartphone owner with enabled messaging, 5) email account, 6) answer 'no' to all questions on the PAR-Q17 health history assessment or are willing to get physician evaluation prior to enrolling and 7) willing to share LoseIt! self-monitoring logs with the investigative team, and attend three assessment visits at the health department. An upper weight limit of 396 pounds reflects the upper measurement capacity for the Withings Body+ Composition smart scale. Per the university Institutional Review Board (IRB) policy, a man with a BMI of 50 or higher will be required to have clinician clearance. We prioritised midlife men in our age selection (over younger men) based on current national overweight/obesity trends and the breadth of current evidence supporting decreasing midlife risk factors (weight loss, physical activity) and increased healthy survival. ${ }^{26-28}$ Exclusion criteria: 1) recently lost $5 \%$ or more body weight, 2) currently taking medications that cause or are influenced by weight loss, 3 ) used weight loss application in the past to lose weight, 4) person from same household is enrolled in study and 5) type I diabetes or type II diabetes with insulin dependence.

\section{Intervention group \\ Self-monitoring}

Lose-It! is a self-monitoring application designed for the general public and includes both a basic (free) and premium (US\$39.99/annually) version. MT+ will receive the Lose-It! premium application, Withings Body+ 
Composition smart scale, daily short message service messages, technology support and a private group discussion board within the application. In addition to the self-monitoring, the premium version permits enhanced customisation of goal setting, application-automated selfmonitoring reminders and customised email reports of self-monitoring trends important in supporting motivation and confidence during periods of behavioural inaction. The smart scale will provide automated recording of weight synchronised to the application, permitting immediate feedback, virtual rewards (ie, badges for achievements) and visual maps of weight trends. The participant will be instructed in how to synchronise the scale with their smartphone at baseline. They will be instructed to weigh themselves daily at home on this scale which will automatically update to their application after each weighing.

\section{Social support}

MT+ participants will be enrolled in a private, closedgroup discussion board created and moderated by the research team. The discussion board will provide social support to MT+ participants while completing the trial to promote long-term success. ${ }^{14} 15$ The discussion board will also provide opportunity for participants to share their self-monitoring experiences, thus providing a mechanism to influence their peer participants to be aware about the value of their own self-monitoring. The groups will be incentivised by a male moderator who will administer peer challenges weekly (see online supplementary appendix I) and will also respond to questions. Participants will also be encouraged to postweekly about their own successful strategies and progress reports related to their self-monitoring for weight loss.

We tested discussion board topics with the $\mathrm{CAB}$ comprising men and women from the region. In addition to this, acceptability feedback about discussion board topics was gathered from rural, male subjects in our preliminary study. ${ }^{5}$ It is noted that the men desired both a combination of both gender-tailored and standardised private discussion board topics. ${ }^{5}$

\section{Text messaging}

A message library (see online supplementary appendix II) will be developed by the team based on messaging content that has demonstrated usefulness for behaviour change ${ }^{29-32}$ and preferred by men. ${ }^{5313334}$ Message content will include a variety of topics including reminders, eating and physical activity behaviours to be enacted and avoided, self-monitoring portion control, strategies for overcoming weight loss barriers and healthy living challenges. Content will be adapted from healthy eating and physical activity promoting resources that include USDA Choose My Plate ${ }^{35}$ and Centers for Disease Control: Physical Activity. ${ }^{36}$ Physical activity includes targeted aerobic physical activity, monitoring of body weight, behaviours needed to sustain weight loss, promoting success and rewarding oneself, preventing failure and avoiding temptations. ${ }^{16} \mathrm{CAB}$ members will inform and review the content of the messages for local relevance prior to dissemination to the participants. An online automatic service (Remind.com) will be used to send the free messages to participants twice per day on Monday, Wednesday and Friday at 08:00 and 11:00 hours, and once per day on Tuesday, Thursday, Saturday and Sunday at 08:00 hours. The delivery time, frequency and number of text messages was based on feedback from midlife, rural male participants in the preliminary study. ${ }^{5}$

\section{Troubleshooting support and re-engagement prompt}

$\mathrm{MT}+$ participants will have access to a 24 hours technology troubleshooting support from the investigative team via phone or text. The participant's food, activity and weight $\log$ will be accessed once weekly by the investigative team to monitor frequency of logging. If dietary intake, physical activity or weight are not logged for $>5$ days, the participant will receive a reminder text and phone call from the assigned graduate level student nurse on the investigative team.

\section{Comparison group}

The MT group of men will receive the Lose-It! basic application. The basic application permits real-time selfmonitoring of eating, physical activity and weight—same as the premium version. The basic version is available for free and is widely accessible by any smartphone user. ${ }^{22}$ MT participants will be asked at baseline to self-monitor their eating, physical activity and weight daily. They will be instructed to weigh daily and $\log$ the result into the application. They will not receive message prompts for self-monitoring, no self-monitoring trend reports and no peer interaction via application-based customised social group. The MT participants will only receive reminders for their assessment visit appointment times.

\section{Technology orientation}

During the baseline visit, the community health worker will train men in both groups to use an assigned application username and password. Hands-on orientation training will be provided about how to use various features of the Lose-It! application (eg, log food intake, measure basic step count, etc). Both groups will receive a paper printed version of an Application User Manual designed and adapted for the study.

\section{Focus groups}

Two focus groups will be held with participants comprising the MT+ group at 6 months postbaseline. A purposive sample of MT+ completers stratified according to their weight loss experience (successful or unsuccessful in achieving the $5 \%$ baseline body weight loss goal) will be sought to solicit their perceptions on the MT+ intervention acceptability and feasibility. ${ }^{37-39}$ A semi-structured interview guide will be used during facilitation of a 90 min discussion led by two co-moderators. Moderatordebriefing and reflexive memos will also be summarised after each interview and audio files will be transcribed 


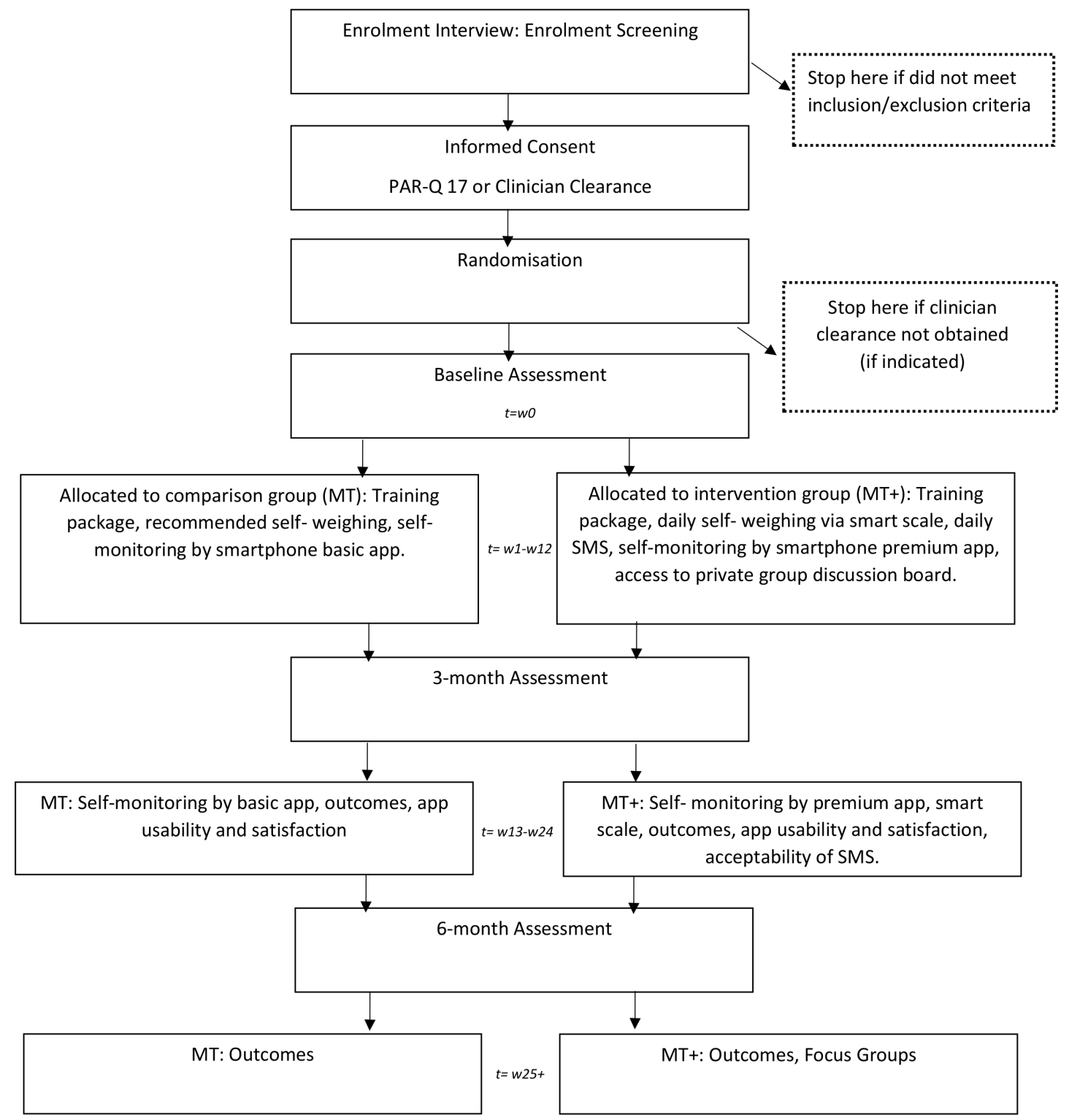

Figure 1 Participant timeline.

verbatim. Collectively, these items will create a decision trail which will serve as an audit demonstrating accountability. ${ }^{40}$ For a detailed participant timeline, see figure 1 .

\section{Recruitment and consent}

Participant recruitment will occur through $\mathrm{CAB}$ and student outreach, Facebook advertising, ClinicalTrials. gov, university webpage, press releases, business bulletin board postings in businesses, community fairs, clinician office outreach and direct referral. Recruitment of minority men, primarily with men who identify as nonwhite Hispanic, will be sought through Spanish-speaking community health workers who already have established local trust. The recruitment period will last for 18 months from June 2018 to November 2019 for trial participants.

Enrolment screening calls will be conducted by a trained graduate student nurse. The physical activity readiness questionnaire (PAR-Q 17) will be used to determine if clinician clearance is required prior to the baseline visit. Thus, informed consent (see online supplementary appendix III) will be obtained prior to its completion during the screening call. Research electronic data capture (REDCap) will permit real-time participant consenting. Study data will be collected and managed using REDCap electronic data capture tools hosted at the university. REDCap is a secure, web-based application designed to support data capture for research studies. When determined eligible, the participant will be invited to participate in the process of informed consent via a REDCap weblink to the secure consenting page sent via text message or email. After live verification of their reading of the consent and answering relevant questions, the participant will provide a wet-signature, immediately verifiable online by the graduate student nurse. The wet signature is a feature in REDCap which allows participants to sign consent forms online in real time, by signing their name with their finger or computer mouse on a 
document as if the participant is using a pen and paper. ${ }^{41}$ This process does not require an additional confirmation procedure ${ }^{42}$ and is customary in REDCap consenting and data collection. ${ }^{41}$ The informed consent document specifies the posting of clinical trial information at ClinicalTrials.gov. A printed copy of the signed consent will be mailed to each participant immediately following conclusion of the enrolment interview.

\section{Randomisation}

Random allocation of group assignment will occur using an allocation schedule created by the project statistician using a random number generator and 'turn randomisation' to ensure equal sample sizes. The outcome assessor will receive a REDCap code and designated application username and password, along with the participant's group assignment to assist participants with successful download and orientation to the application version.

\section{Outcomes, measurements, data management and analysis Outcomes \\ Feasibility and acceptability}

Feasibility and acceptability will be evaluated using number of men recruited and retained, $\mathrm{CAB}$ member feedback, application use and evaluative focus group feedback from intervention participants.

\section{Clinical outcomes}

Preliminary efficacy of 3-month and 6-month weight loss ( $\mathrm{kg}$ and \% body weight using a standard clinic scale) and improved dietary and physical activity behaviours (survey and data from Lose-It! Application (kcal/day, steps/day) will be examined. The Tanita Scale (TBF-215) will be used to measure height, weight and $\mathrm{BMI}^{43}$ at the baseline, 3-month and 6-month assessment visits. Weekly, the graduate student nurse will also log into each participant's Lose-It! account and export participant-logged weights. Fruit and vegetable consumption will be measured using the BRFSS Fruit and Vegetable Dietary Intake Module (six items). ${ }^{44}$ The BRFSS Physical Activity Questionnaire will also be used to measure self-reported physical activity of the participants. ${ }^{44}$ To measure the sugar sweetened beverage intake, the Brief Questionnaire to Assess Beverage Intake (BEVQ-15) ${ }^{45}$ will be used. Participant's 'weekly summaries' will also be exported from the app which includes daily food log, physical activity, weights and total daily step count.

\section{Community capacity}

Community capacity will be assessed using a community capacity evaluation survey that has been tested and applied with other rural US communities for obesity prevention. ${ }^{46}$ Descriptive content analysis will evaluate intervention acceptability and contextual sensitivity.

\section{Measures}

Figure 2 outlines the outcome measures. Measures at 3 months and 6 months will be compared with baseline. Additionally, health history, demographics, blood

\begin{tabular}{|l|l|l|}
\hline \multicolumn{3}{|c|}{ Measure Description (Data Collection Time Points) } \\
\hline Primary Outcomes: Body Mass Index and Weight & $\begin{array}{l}\text { Baseline, 3 months, and 6 } \\
\text { months }\end{array}$ \\
\hline Body mass index & Tanita Scale (TBF-215) & $\begin{array}{l}\text { Baseline, 3 months, and 6 } \\
\text { months }\end{array}$ \\
\hline Weight & $\begin{array}{l}\text { In-person measurement of } \\
\text { weight by the Tanita Scale } \\
\text { (TBF-215) } \\
\text { WithingsO Body+ Body } \\
\text { Composition Smart Scale (MT+) }\end{array}$ & $\begin{array}{l}\text { Daily measure (Recommended), } \\
\text { Weekly average computed }\end{array}$ \\
Self-reported weight (MT) & $\begin{array}{l}\text { Daily (Recommended), Weekly } \\
\text { average computed }\end{array}$ \\
\hline Secondary Outcomes: Diet and Physical Activity & $\begin{array}{l}\text { Baseline, 3 months, and 6 } \\
\text { months }\end{array}$ \\
\hline $\begin{array}{l}\text { Fruit and Vegetable } \\
\text { Servings }\end{array}$ & $\begin{array}{l}\text { BRFSS-Fruit and Vegetable } \\
\text { Dietary Intake module }\end{array}$ \\
\hline $\begin{array}{l}\text { Sugar-Sweetened/ } \\
\text { Total Beverage Energy } \\
\text { Intake }\end{array}$ & $\begin{array}{l}\text { Brief Questionnaire to Assess } \\
\text { Beverage Intake (BEVQ-15) }\end{array}$ & $\begin{array}{l}\text { Baseline, 3 months, and 6 } \\
\text { months }\end{array}$ \\
\hline Physical Activity & $\begin{array}{l}\text { BRFSS- Physical activity } \\
\text { module }\end{array}$ & $\begin{array}{l}\text { Baseline, 3 months, and 6 } \\
\text { months }\end{array}$ \\
\hline $\begin{array}{l}\text { Report of daily log of } \\
\text { dietary intake, physical } \\
\text { activity, and weight }\end{array}$ & $\begin{array}{l}\text { Weekly summary downloaded } \\
\text { from app which includes } \\
\text { participants self- report of } \\
\text { dietary intake, physical activity, } \\
\text { weight, and steps per day }\end{array}$ & $\begin{array}{l}\text { Weekly reports exported during } \\
\text { study }\end{array}$ \\
\hline
\end{tabular}

Figure 2 Figure 2Outcome Measures.

pressure, pulse rate, Comfort with Technology survey and health information technology usability evaluation scale (health ITUES) will be collected for further analysis of participants' profile and outcomes (figure 2).

\section{Height, weight and BMI}

The Tanita TBF-215 Body Composition Analyzer will be used to measure the participant's height, weight and BMI following their manual guidelines. ${ }^{43}$ The participant will be asked to remove shoes, socks, belt and empty pockets. The participant will be asked to stand up straight so height can be accurately measured. After height is measured, the participant will be asked to step off the scale, the scale will be zeroed and the participant will step back on the scale to measure weight. Once the height and weight is confirmed, a paper copy of the participants' values will print out with the calculated BMI.

\section{Food and beverage intake}

To establish a baseline of food and beverage intake, the BRFSS Fruit and Dietary Intake Survey and the BEVQ-15 will be administered to participants at time points as shown in figure $2 .{ }^{3645}$ The consumption of these are indicators of a healthy overall diet, ${ }^{636}$ and given the resourceconstrained nature of feasibility studies we wanted to reduce participant's burden for data collection. Fruit and vegetable intake is an indicator used nationally to monitor and establish benchmarks of a healthy overall diet. $^{36}$ The BRFSS, in most states, is the only source of uniform nutritional data for adults. ${ }^{36}$ Sugar-sweetened beverage consumption is a major source of calories that has received increasing attention in recent years as playing a role in the obesity epidemic. ${ }^{45} 4748$ Additionally, a lack of fruit and vegetable intake is more common among men ${ }^{49}$ and rural residents, ${ }^{50}$ which relates to our study population.

Additional information regarding fruit and vegetable and sugar-sweetened beverage intake will be exported from the application logs and analysed. Each week, the graduate student nurse will log into the participants' web 
version of the application to retrieve the 'weekly summary' of meals/day logged and average calories/day/week and export the data to participants' ID-labelled file.

\section{Physical activity}

The BRFSS Physical Activity Questionnaire will be used to gather participants' self-report of physical activity. ${ }^{44}$ Additionally, like the food intake, each week the graduate student nurse will retrieve from the application the 'weekly summary' of physical activity and total daily step count.

\section{Blood pressure and pulse rate}

The ADC e-sphyg 29002 Automatic Sphygmomanometer will be used to measure blood pressure of the participants. This model was selected due to specifications $\pm 3 \mathrm{~mm} \mathrm{Hg}$ accuracy of cuff pressure consistent with acceptable standards, current acceptability of an oscillometric blood pressure unit in field and clinical areas ${ }^{51}$ and prior successful prior use in a large clinical trial. ${ }^{52}$ In addition, we did a test-retest before using this unit and determined the auto feature was appropriate for field trials such as ours. Routine checks for calibration will be conducted every 6 months as recommended by the manufacturer.

The participant will be asked to wear loose clothing or a short sleeve shirt, to avoid caffeine, intensive exercise and smoking for at least $30 \mathrm{~min}$ before measurement. ${ }^{52}$ The participant will be instructed to not talk during the rest period or during measurement. The man's arm will be placed on a desk or table so that the middle of the arm is at the level of the heart. The outcome assessor will line up the cuff mark 'artery' over the individual's brachial artery. For $5 \mathrm{~min}$, the participant will quietly sit in a chair (feet on floor, back supported) and rest without talking. After obtaining a blood pressure, the participant will be asked to raise his arm for $10 \mathrm{~s}$ and wait another $30 \mathrm{~s}$. Then the blood pressure procedure will be repeated with measurements spaced about 1 min apart. The procedure will be repeated until two readings are within $5 \mathrm{~mm} \mathrm{Hg}$ and average the two values together. Two resting pulse rates will also be obtained using the blood pressure cuff and these values will be averaged together and recorded. ${ }^{53}$

\section{Health history and demographics}

A brief health history survey will be administered at baseline. A generic demographics form was also administered to participants at baseline.

\section{Technology experience}

Technology experience will be evaluated with the Comfort with Technology survey ${ }^{54}$ (baseline), and the Technology Feasibility and Acceptability survey, which was adapted from the health-ITUES ${ }^{55}$ (3 and 6 months). The Comfort with Technology survey asks questions related to comfort, frequency and purpose of technology use. ${ }^{54}$ The modified health-ITUES evaluates technology usefulness. ${ }^{55}$

\section{Data management}

The outcome assessor will receive a participant-specific REDCap code to enter assessment information. To encourage participant retention, the graduate student nurse will contact MT+ group participants if they fail to $\log$ their eating, activity or weight in the Lose-It! application for $>5$ days. If the participant states he cannot $\log$ daily, the graduate student nurse will document reasons and encourage participant to post as often as he can.

\section{Analysis}

Aim 1: MT+ intervention feasibility and acceptability

Aim 1 will be evaluated through: 1) participation rates including number of men recruited and randomised over a 6-month period; 2) participant retention rates; 3) feasibility, usability, satisfaction ratings; 4) application logs of $\mathrm{MT}+$ and 5) evaluative focus group feedback. Descriptive statistics will be calculated on all variables, including frequencies and percentages for recruitment/retention, demographic and categorical variables. To determine feasibility of recruitment, a rate will be measured in the time it takes to enrol 80 participants. To determine feasibility of retention, we used a threshold retention of $70 \%$ which is similar to studies of weight loss in men. ${ }^{13},{ }^{56}$ Feasibility, usability and satisfaction ratings will be measured from modified health-iTUES, which was originally validated by authors to measure technology usability, but has further been validated to be used as a customisable technology survey. ${ }^{57}$ Means and SD will be calculated for all continuous variables and measures at each time point. Feasibility and acceptability analyses for aim 1 are largely descriptive, as we will be assessing participation rates and percentages of eligible men and which recruitment methods were the most effective.

Qualitative content analysis ${ }^{40}$ will guide interpretation of the focus group findings. The interview transcript and reflective memos taken during each focus group will comprise one unit of analysis for within/across case comparison. The topics outlined by the interview guide will be extracted and organised and transcripts read for substantive coding. Data 'facts' will be organised under a priori coding categories. The categories are named a priori because they are built into the interview guide questions. A data 'fact' will be defined as those data elements that recurred in the interview without lack of consensus or were least participant to errors in inference ${ }^{58}$ All data provided in a response to each question will be coded together. Incomplete, competing or alternative topics that present in the discussion but were not identified a priori will be aggregated and examined to determine their fit with the purpose.$^{59} \mathrm{~A}$ data matrix will be used to display the coded data to search for patterns across coding categories. The principal investigator and graduate student nurse will return to the data to explore patterns further, supporting iterative analysis. Data categories will be re-contextualised into an account that makes sense for the entire study's data set. Meaning, the findings are integrated to provide new understanding 
or explanation to the interpretation of the intervention outcome data. ${ }^{59}$ Peer-debriefing and audit checking will occur weekly across the analysis to assure accuracy of the findings.

Aim 2: variability of outcome measures and sample size estimation Descriptive statistics of participants' profiles for outcomes by time point and stratified by intervention group will be reported. The proportion of participants meeting the clinically meaningful threshold of $5 \%$ wt loss over the course of the study will also be reported. A maximum likelihood estimation method (ie, mixed models) will be used in order to use all available data. Outcome variables that are not normally distributed will either be transformed or assessed with non-parametric methods. An independent group t-test will be used to assess overall weight loss at follow-up solely to estimate an effect size (Cohen's d for weight loss between groups) for sample size estimation for a future large trial.

\section{Aim 3: indicators of community capacity}

Multiple indicators of community capacity will be used to evaluate support for the weight loss intervention applying best practice recommendations: 1) CAB-assessed community capacity change via survey report, ${ }^{46}$ participation level of $\mathrm{CAB}$ members (ie, number of attended meetings, activities, resources allocated, partnership linkages), member attrition with reasons, perceived benefits/skills gained, barriers and facilitators of retention, proposed strategies to increase retention, 2) student support in the outreach and recruitment will be tracked via number of hours of participation and partnership linkages.

\section{Data monitoring, auditing and harm}

Each participant will be given a unique study identifier, all protected health information will be masked, and REDCap data exports will be limited to the principal investigator and the project statistician for generating reports and the conduct of statistical data analysis. Safety monitoring will be conducted monthly by the principal investigator, study statistician and independent data safety monitor. Per university policy, all serious adverse events (AEs) and unintended effects of the intervention will be reported to the university IRB and the independent data safety monitor (IDSM) within 2 days after the principal investigator is notified of the AEs. The technology safety report will include troubleshooting requests from participants, re-engagement attempts for participants who were not logging and any technology-related protocol violations. The enrolment safety report includes new enrolment counts, subject withdrawals, protocol violations, AEs and preliminary outcomes.

\section{ETHICS AND DISSEMINATION}

This protocol, including consent forms, has been approved by the University of Nebraska Medical Center IRB (UNMC IRB\# 594-17-EP). All protocol amendments will be communicated immediately to the IRB, DSMP, ClinicalTrials.gov, $\mathrm{CAB}$, participants and funder. All participants will be informed of their right to confidentiality right to leave the trial at any point without loss of those benefits to which they were entitled. All data will be retained in HIPPA compliant REDCap database. REDCap at UNMC is supported by Research IT Office funded by Vice Chancellor for Research.

\section{Access to data}

The principal investigator, study statistician and the designated IDSM will have access to the final trial dataset. All proposed study-specific case report forms for data collection will be coded by the participants' unique study ID and maintained in REDCap. All data and other personal health information will be removed from the study database on completion of the study.

\section{Ancillary and post-trial care}

Post-trial care is not anticipated as this trial is classified as a low-risk intervention. Participants who express need for assistance will be informed of the UNMC support services and assisted in contacting them.

\section{Dissemination policy}

Trial is registered at ClinicalTrials.gov, Identifier: NCT03329079. De-identified summary results will be posted to ClinicalTrials.gov for public access and disseminated in scientific forums and to the local rural communities.

Acknowledgements The authors would like to acknowledge the Rural Men's Health Study Community Advisory Board and UNMC Northern Division student nurses for contributions to recruitment outreach and contextual tailoring of the intervention.

Contributors CE is PI directing all study components. KAK is the statistician who co-leads data management. FAB, AMY and KES develop and manage the mobile technologies. PH provides protocol oversight, assessment fidelity planning and training, JM conducts enrolment screening, recruitment, $C A B$ and focus group moderation. CHP advises ethics board and protocol adherence. Manuscript drafted by CE, KES and all authors revised-approved the final version. All authors contributed substantially to study design and protocol conduct.

Funding Study supported by the National Institute of Nursing Research of the National Institute of Health under award number R15NR017522.

Competing interests None declared.

Patient consent for publication Not required.

Provenance and peer review Not commissioned; externally peer reviewed.

Open access This is an open access article distributed in accordance with the Creative Commons Attribution Non Commercial (CC BY-NC 4.0) license, which permits others to distribute, remix, adapt, build upon this work non-commercially, and license their derivative works on different terms, provided the original work is properly cited, appropriate credit is given, any changes made indicated, and the use is non-commercial. See: http://creativecommons.org/licenses/by-nc/4.0/.

ORCID iD

Christine M Eisenhauer http://orcid.org/0000-0002-8591-7162

\section{REFERENCES}

1 Hales C, Carroll M, Fryar C, et al. Prevalence of obesity among adults and youth: United States, 2015-2016. Hyattsville, MD: National 
Center for Health Statistics, 2017. https://www.cdc.gov/nchs/ products/databriefs/db288.htm

2 Ogden CL, Carroll MD, Kit BK, et al. Prevalence of childhood and adult obesity in the United States, 2011-2012. JAMA 2014;311:806-14.

3 Meit M, Knudson A, Gilbert T, et al. The 2014 update of the ruralurban chartbook. Bethesda. Rural Health Reform Policy Research Center, 2014

4 Shelton JB, Rajfer J. Androgen deficiency in aging and metabolically challenged men. Urol Clin North Am 2012;39:63-75.

5 Eisenhauer CM, Hageman PA, Rowland S, et al. Acceptability of mHealth technology for self-monitoring eating and activity among rural men. Public Health Nurs 2017;34:138-46.

6 Lundeen E, Park S, Liping P, et al. Obesity prevalence among adults living in metropolitan and nonmetropolitan countries-Unitied states. Morbidity and Mortality Weekly Report 2016:653-8.

7 Guo Z, Jiang Y, Huffman SK. Agricultural mechanization and BMI for rural workers: a field experiment in China. Economics Working Papers 2018

8 Pickett W, King N, Lawson J, et al. Farmers, mechanized work, and links to obesity. Prev Med 2015;70:59-63.

9 Han M, Lee E. Effectiveness of mobile health application use to improve health behavior changes: a systematic review of randomized controlled trials. Healthc Inform Res 2018;24:207.

10 Beleigoli A, Andrade A, Cançado A, et al. The impact of web-based digital health interventions on weight loss and lifestyle habits changes in overweight and obese adults: a systematic review and meta-analysis (Preprint). Journal of Medical Internet Research 2017;21.

11 Fortuin J, Salie F, Abdullahi LH, et al. The impact of mHealth interventions on health systems: a systematic review protocol. Syst Rev 2016;5:200.

12 Anderson-Lewis C, Darville G, Mercado RE, et al. mHealth technology use and implications in historically underserved and minority populations in the United States: systematic literature review. JMIR Mhealth Uhealth 2018;6:e128.

13 Robertson C, Avenell A, Stewart F, et al. Clinical effectiveness of weight loss and weight maintenance interventions for men: a systematic review of men-only randomized controlled trials (the ROMEO project). Am J Mens Health 2017;11:1096-123.

14 Neumark-Sztainer D, Sherwood NE, French SA, et al. Weight control behaviors among adult men and women: cause for concern? Obes Res 1999;7:179-88.

15 Lemon SC, Rosal MC, Zapka J, et al. Contributions of weight perceptions to weight loss attempts: differences by body mass index and gender. Body Image 2009;6:90-6.

16 French SA, Jeffery RW, Wing RR. Sex differences among participants in a weight-control program. Addict Behav 1994;19:147-58.

17 Lovejoy JC, Sainsbury A, Stock Conference 2008 Working Group. Sex differences in obesity and the regulation of energy homeostasis. Obes Rev 2009;10:154-67.

18 Klitzman P, Armstrong B, Janicke DM. Distance as a predictor of treatment attendance in a family based pediatric weight management program in rural areas. J Rural Health 2015;31:19-26.

19 Hiebert B, Leipert B, Regan S, et al. Rural men's health, health information seeking, and gender identities: a conceptual theoretical review of the literature. Am J Mens Health 2018;12:863-76.

20 Graham LJ, Connelly DM. "Any movement at all is exercise": a focused ethnography of rural community-dwelling older adults' perceptions and experiences of exercise as self-care. Physiother Can 2013:65:333-41.

21 Whitehead AL, Sully BGO, Campbell MJ. Pilot and feasibility studies: is there a difference from each other and from a randomised controlled trial? Contemp Clin Trials 2014;38:130-3.

22 Zuidgeest MGP, Goetz I, Groenwold RHH, et al. Series: pragmatic trials and real world evidence: paper 1. Introduction. J Clin Epidemiol 2017;88:7-13.

23 Lancaster GA, Dodd S, Williamson PR. Design and analysis of pilot studies: recommendations for good practice. J Eval Clin Pract 2004;10:307-12.

24 Browne RH. On the use of a pilot sample for sample size determination. Stat Med 1995;14:1933-40.

25 National Institutes of Health. Academic research enhancement award (parent R15) PA-16-200. In: Department of health and human services Part 1 overview information. National Institutes of Health, 2017.

26 Robsahm TE, Heir T, Sandvik L, et al. Changes in midlife fitness, body mass index, and smoking influence cancer incidence and mortality: a prospective cohort study in men. Cancer Med 2019;8:4875-82.
27 Strandberg TE, Sirola J, Pitkälä KH, et al. Association of midlife obesity and cardiovascular risk with old age frailty: a 26-year followup of initially healthy men. Int J Obes 2012;36:1153-7.

28 Holme I, Tonstad S. Survival in elderly men in relation to midlife and current BMl. Age Ageing 2015;44:434-9.

29 Shapiro JR, Koro T, Doran N, et al. Text4Diet: a randomized controlled study using text messaging for weight loss behaviors. Prev Med 2012;55:412-7.

30 Shaw RJ, Bosworth HB, Hess JC, et al. Development of a theoretically driven mHealth text messaging application for sustaining recent weight loss. JMIR Mhealth Uhealth 2013;1:e5.

31 Patrick K, Raab F, Adams MA, et al. A text message-based intervention for weight loss: randomized controlled trial. J Med Internet Res 2009;11:e1.

32 Gerber BS, Stolley MR, Thompson AL, et al. Mobile phone text messaging to promote healthy behaviors and weight loss maintenance: a feasibility study. Health Informatics J 2009;15:17-25.

33 Joo N-S, Kim B-T. Mobile phone short message service messaging for behaviour modification in a community-based weight control programme in Korea. $J$ Telemed Telecare 2007:13:416-20.

34 Lee D, Moon J, Kim YJ, et al. Antecedents and consequences of mobile phone usability: linking simplicity and interactivity to satisfaction, trust, and brand Loyalty. Inf Manage 2015;52:295-304.

35 United States Department of Agriculture. Dietary guidlines for Americans online: USDA, 2018. Available: https://www. choosemyplate.gov/dietary-guidelines

36 Centers for Disease Control and Prevention. Surveillance of fruit and vegetable intake using the behavioral risk factor surveillance system, 2015. Available: https://www.cdc.gov/brfss/pdf/fruits_vegetables.pdf

37 Hydén L-C, Bülow PH. Who's talking: drawing conclusions from focus groups-some methodological considerations. Int J Soc Res Methodol 2003;6:305-21.

38 Rodgers BL, Cowles KV. The qualitative research audit TRAIL: a complex collection of documentation. Res Nurs Health 1993;16:219-26.

39 Mannell J, Davis K. Evaluating complex health interventions with randomized controlled trials: how do we improve the use of qualitative methods? Qual Health Res 2019;29:623-31.

40 Hsieh H-F, Shannon SE. Three approaches to qualitative content analysis. Qual Health Res 2005;15:1277-88.

41 Haussen DC, Doppelheuer S, Schindler K, et al. Utilization of a smartphone platform for electronic informed consent in acute stroke trials. Stroke 2017;48:3156-60.

42 Civelek ME, Uca N, Cemberci M. eUCP and electronic commerce investments: E-signature and paperless foreign trade. Eurasian Academy of Sciences, Eurasian Business \& Economics Journal 2015;3.

43 Tsui EY, Gao XJ, Zinman B. Bioelectrical impedance analysis (BIA) using bipolar foot electrodes in the assessment of body composition in type 2 diabetes mellitus. Diabet Med 1998;15:125-8.

44 Brownson RC, Jones DA, Pratt M, et al. Measuring physical activity with the behavioral risk factor surveillance system. Med Sci Sports Exerc 2000;32:1913-8.

45 Hedrick VE, Savla J, Comber DL, et al. Development of a brief questionnaire to assess habitual beverage intake (BEVQ-15): sugarsweetened beverages and total beverage energy intake. $J$ Acad Nutr Diet 2012;112:840-9.

46 Zoellner J, Hill JL, Brock D, et al. One-Year mixed-methods case study of a Community-Academic Advisory board addressing childhood obesity. Health Promot Pract 2017;18:833-53.

47 Lundeen EA, Park S, Pan L, et al. Daily intake of sugarsweetened beverages among US adults in 9 states, by state and sociodemographic and behavioral characteristics, 2016. Prev Chronic Dis 2018;15:E154-E54.

48 Malik VS, Schulze MB, Hu FB. Intake of sugar-sweetened beverages and weight gain: a systematic review. Am J Clin Nutr 2006;84:274-88.

49 Singh GM, Micha R, Khatibzadeh S, et al. Global, regional, and national consumption of sugar-sweetened beverages, fruit juices, and milk: a systematic assessment of beverage intake in 187 countries. PLoS One 2015;10:e0124845.

50 Sharkey JR, Johnson CM, Dean WR. Less-healthy eating behaviors have a greater association with a high level of sugar-sweetened beverage consumption among rural adults than among urban adults. Food Nutr Res 2011;55:5819.

51 Pickering TG, Hall JE, Appel LJ, et al. Recommendations for blood pressure measurement in humans and experimental animals. Hypertension 2005;45:142-61.

52 Hageman PA, Pullen $\mathrm{CH}$, Hertzog $\mathrm{M}$, et al. Web-Based interventions alone or supplemented with peer-led support or professional email counseling for weight loss and weight maintenance in 
women from rural communities: results of a clinical trial. $J$ Obes 2017:2017:1-21.

53 Perloff D, Grim C, Flack J, et al. Human blood pressure determination by sphygmomanometry. Circulation 1993:88:2460-70.

54 Eisenhauer Cet al. Partnering with rural farm women for participatory action and ethnography. Online Journal of Rural Nursing and Health Care 2016;16:196-216.

55 Yen P-Y, Wantland D, Bakken S. Development of a customizable health it usability evaluation scale. AMIA Annu Symp Proc 2010;2010:917-21.

56 Morgan PJ, Callister R, Collins CE, et al. The SHED-IT community trial: a randomized controlled trial of internet- and paper-based weight loss programs tailored for overweight and obese men. Ann Behav Med 2013;45:139-52.

57 Yen P-Y, Sousa KH, Bakken S. Examining construct and predictive validity of the Health-IT usability evaluation scale: confirmatory factor analysis and structural equation modeling results. J Am Med Inform Assoc 2014;21:e241-8.

58 Saldaña J. The coding manual for qualitative researchers. 3rd edn. Thoursand Oaks: Sage, 2015.

59 Liberato SC, Brimblecombe J, Ritchie J, et al. Measuring capacity building in communities: a review of the literature. BMC Public Health 2011:11:850. 